\title{
Visualising Java Coupling and Fault Proneness
}

\author{
P. Rosner ${ }^{1}$, M. Child ${ }^{1}$ and S. Counsell ${ }^{2}$ \\ ${ }^{I}$ Department of Informatics, Faculty of Business, London South Bank University, London, U.K. \\ ${ }^{2}$ Department of Information Systems, Brunel University, Uxbridge, U.K. \\ \{rosnerpe,childm\}@lsbu.ac.uk,steve.counsell@brunel.ac.uk
}

Keywords: Object-oriented, Coupling, Metrics, Visualisation.

\begin{abstract}
In this paper, a tool is described for visualising the Coupling Between Objects (CBO) metric for Java systems, decomposing it into coupling collaborators and using colour to denote the object-oriented mechanisms at work for each couple. The resulting visualisation is also envisaged to be useful for general program comprehension and is integrated into Java development in the Eclipse IDE. Evidence is also given that the visualisation may help detect classes tending to be less fault-prone than would be expected from inspection of their $\mathrm{CBO}$ values alone.
\end{abstract}

\section{INTRODUCTION}

The metric Coupling Between Objects or CBO (Chidamber and Kemerer, 1994) has been shown to correlate with quality indicators such as faultproneness (Olague et al., 2007). CBO for a class is based on the number of distinct collaborators either accessed from (fan-out) or accessing (fan-in) the class. However, if only the raw CBO value is available, the program code needs to be examined manually to discover $\mathrm{CBO}$ collaborators, and to verify that a high $\mathrm{CBO}$ value for a particular class is indeed harmful. CouplingViz, a visualisation tool for Java systems addresses this issue. It decomposes the CBO for a class into its collaborator classes and interfaces, allowing the developer to delve more deeply into cases of high CBO values without necessarily needing to examine program code.

More generally, knowing the collaborators of each class has been considered an important aspect of understanding a system (Biddle et al., 2002). CouplingViz therefore also takes on a more general program comprehension and navigation role beyond merely focusing on cases of high coupling.

The tool provides a map of all classes and interfaces in a system. The map indicates CBO visually and allows interactive selection of a class or interface to view its name, numerical CBO value, coupling collaborators and, via colour, associated object-oriented coupling categories.
Selection can either be carried out directly on a class representation in the visualisation, or else by selecting the corresponding class in the Eclipse IDE. Automatic navigation to program code for a selection is also provided. This corresponds to operating at different levels of detail to help to understand and navigate large and complex systems - from the bird's eye view, through the intermediate '1000 foot view' above the code (Doernenburg, 2009), down to the code itself if necessary. Moving through these different levels, some of the questions that can be addressed using CouplingViz are:

- Q1: What is the usage pattern for the different types of object-oriented mechanisms involved in class interaction in the system?

- Q2: What are the collaborators for a particular class?

- Q3a: For a particular class is there a pattern to the way it interacts with its collaborators?

- Q3b: Can a developer predict that a class with such a pattern is less likely to be fault-prone than would otherwise be expected from CBO size?

- Q4: What members (method calls, fields) are accessed by a particular class on a collaborator?

- Q5: Which member accesses either to 'self' inside a particular class, or to a target collaborator outside it, are resolved via inheritance in an ancestor class? 


\section{DESCRIPTION OF CouplingViz}

CouplingViz is a plugin to Eclipse to display both fan-out and fan-in CBO for a Java project. Here, we use, as an example, the Web templating open-source Java system Velocity.

A detailed 'bar view' of fan-out is shown in Figure 1. Vertical bars represent its 262 classes and interfaces. The coupling value of each class is indicated by the depth of each bar, with the segments on the bar being coloured according to categories denoting different object-oriented mechanisms as described below. The depth of each segment is proportional to the number of couples for each category. Where the total vertical depth is greater than the inter-row gap, a horizontal bar is displayed at its base whose depth is proportional to the excess. A white dot indicates a class with no fanout coupling. Inner classes are narrower than regular classes. Interfaces are shown as circles. Package boundaries are shown as dashed vertical lines above the rows

Figure 2 shows the default birds-eye view of the system. Here rows of square boxes and circles respresent the classes and Java interfaces respectively. Classes and inner classes with no fanout coupling are shown as shallow. The amount of fan-out coupling for a class or inner class is indicated by the shade of grey of its corresponding box. Classes and interfaces can be 'moused' over for display of their names and their $\mathrm{CBO}$ values.

The colour scheme is shown on the legend in Figures 1 and 2. Blue indicates direct coupling, meaning that at least one method call, constructor call or field access from the source class is resolved in the target. Yellow indicates local inheritance coupling: the coupled target is an ancestor class of the source class, containing at least one of the methods called or fields accessed from within the source class. Purple means foreign inheritance coupling: at least one method call or field access from the source class to a target has its resolved destination not in the target, but rather, via inheritance, in one of the target's ancestors. Green indicates interface coupling: at least one call from the source is to an abstract method in the target (either an interface or abstract class).

The bar view in Figure 1 shows the pattern of the coupling categories at work in Velocity through the incidences of the different colours. Whilst direct coupling (blue) is predominant, interface coupling (green) also has a significant presence, particularly in one package. The presence of local inheritance coupling (yellow) is a little lower, and foreign inheritance coupling (purple) even lower. This type of analysis shows how the visualisation allows us to address Q1.

In the bird's eye view in Figure 2, the class Parse has been selected to display fan-out. This can either be selected by clicking on its representation in the visualisation, or else as in this case, by selecting the class in the Eclipse package explorer. The coupling targets are displayed and each can be 'moused' over showing its name (addressing Q2) and the number of methods/fields accessed by the source on the target.

Arrows appear above the targets coloured according to the coupling categories. Figure 2 also shows the outcome of the following: the target class EventHandlerUtil with a blue arrow, indicating direct coupling, has been selected, the start of the class EventHandlerUtil has been jumped to in the Eclipse code pane and the name of the single method accessed from Parse has displayed in a pop-up list (addressing Q4) in corresponding blue text; the method has then been selected, and its code is now jumped to in the Eclipse code pane.

In Figure 3, the ancestor target Directive has been clicked, the start of the code for this class has appeared in the Eclipse code pane, and a list has popped up showing the names of four methods accessed from within Parse. The yellow colour of the text corresponds to the yellow of the arrow indicating local inheritance coupling for these accesses (addressing Q5). Then the method postRender () has been selected and the code for it has been jumped to in the Eclipse code pane.

In Figure 4 all of the 40 targets of the source class BaseVisitor are coupled to it via foreign inheritance coupling (purple arrows). One of these targets ASTIfStatement has been clicked and a single method appears in the pop up list, also in purple (also addressing Q5). Clicking on this method shows a purple link to the destination ancestor class SimpleNode where the method call resolves, and on which a large purple arrow flashes. The code for the method in SimpleNode is jumped to in the Eclipse code pane. From the visualisation, it is clear that all the targets are in a single package. Clicking on all of the targets reveals the same single method call from BaseVisitor and the destination to this call is revealed to be the same ancestor class SimpleNode. Despite the high coupling value, the visualisation has helped reveal a designed ordered relationship between the source, targets and common inherited destination (addressing Q3a) - the names of the classes also helping in detecting the design. It is likely that the high $\mathrm{CBO}$ value here would not be 


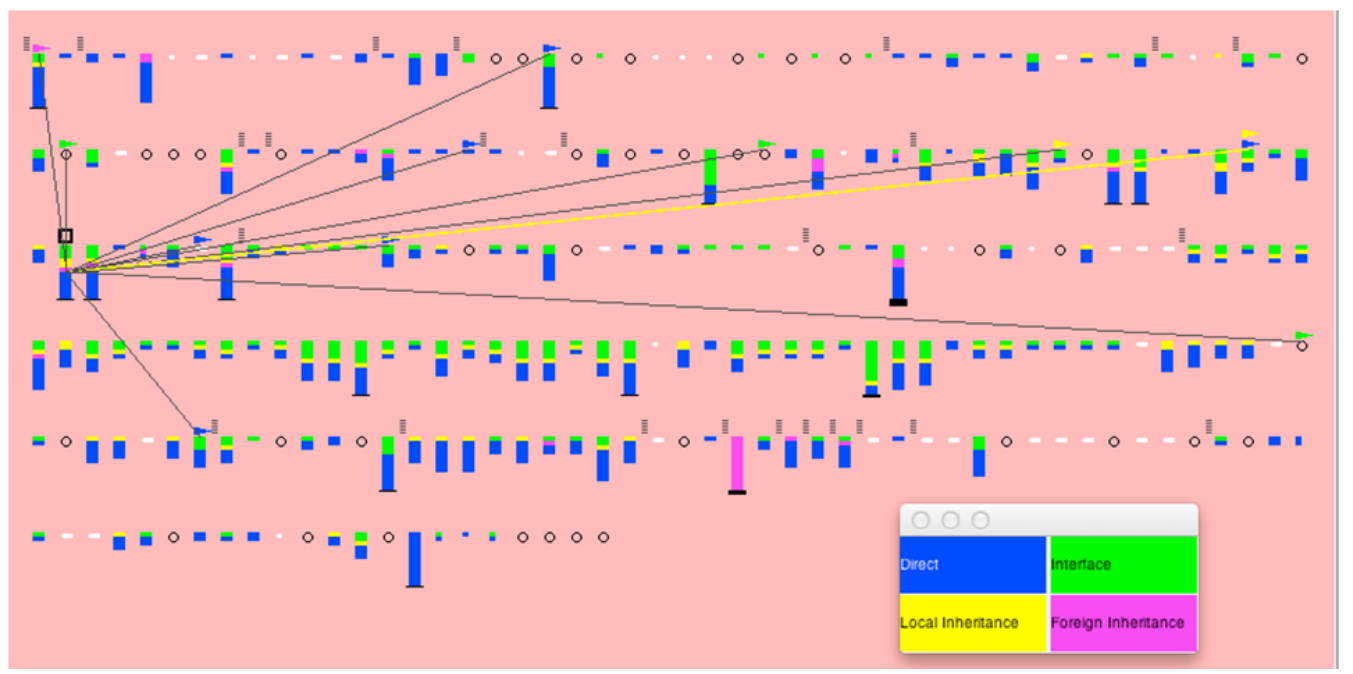

Figure 1: fan-out, bar view - class Parse selected.

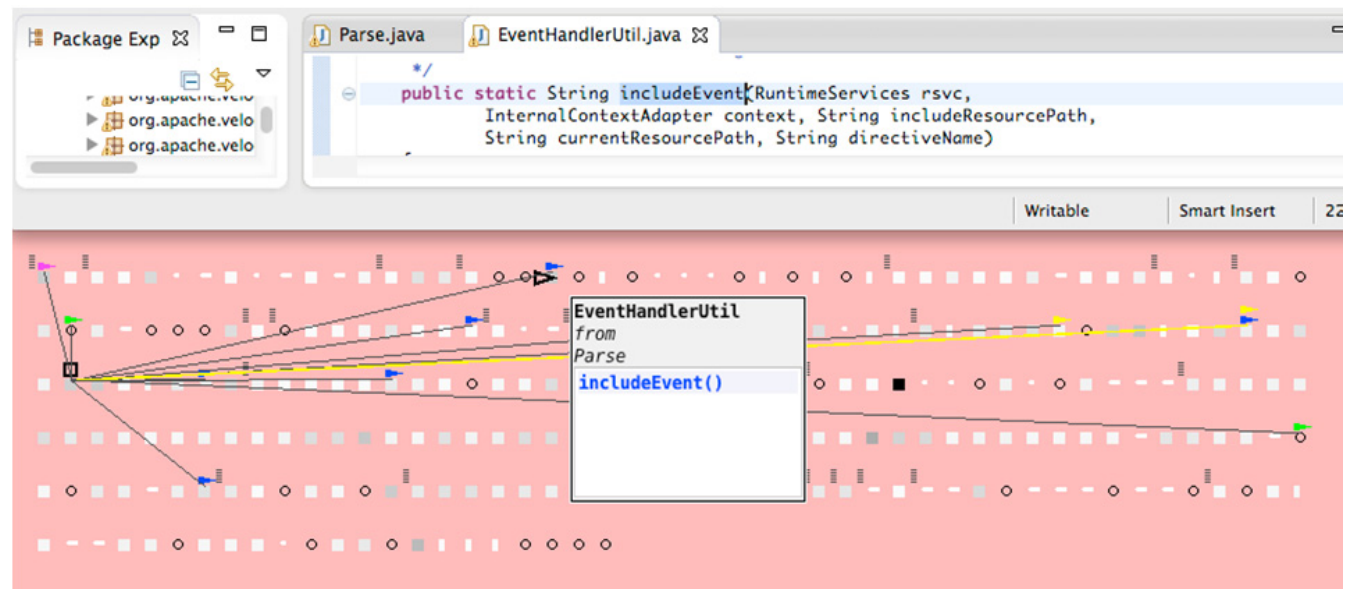

Figure 2: fan-out, birds-eye view, direct coupling from Parse to EventHandlerUtil.

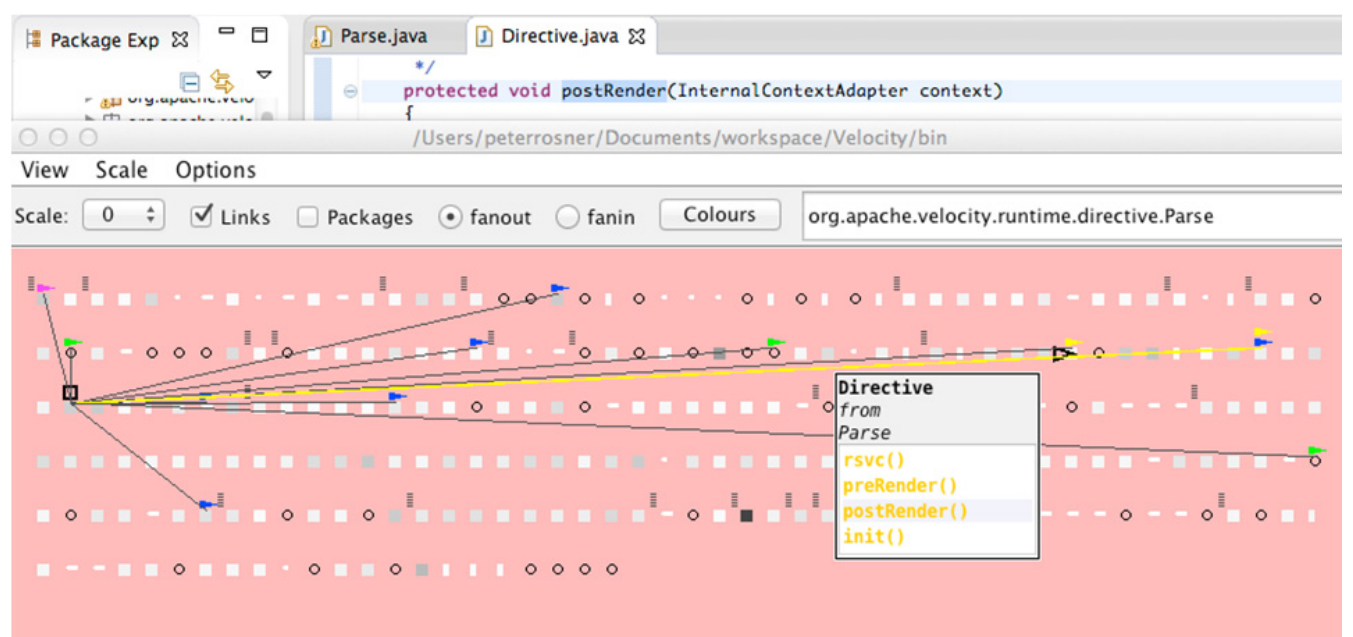

Figure 3: fan-out, inheritance coupling from Parse to Directive. 
seen as harmful.

Figures 5 and 6 demonstrate the difference between fan-out and fan-in coupling. Figure 5 shows the fan-out coupling from the class Parser. Figure 6 shows fan-in coupling for the same class. The members in the target accessed can be obtained by clicking on a source class indicated with coloured arrows above it. In this case by clicking on the source class ASTDirective, the list of methods it calls on Parser is displayed. The method isDirective () has then been selected and the code in Parser for this method is displayed in the Eclipse code pane. It can be seen by comparing Figures 5 and 6 how the visualisation easily shows the difference between the extent of fan-out and fanin. In the case of Parser, fan-out predominates.

\section{NATURE OF THE VISUALISATION}

The CouplingViz overview is a small multiples visualisation, allowing the simultaneous display of the differences between individual classes and the range of values present in the system (Tufte, 1990). At the highest level, this depicts the CBO of each class. The visualisation then provides multiple levels of further information to be revealed using the details on demand paradigm (Shneiderman, 1996). In the decomposed overview, further information is revealed about the nature of the coupling involved, allowing classes with equivalent amounts of $\mathrm{CBO}$ to be contrasted against each other. The selection of individual source classes reveals the specific collaborator classes which are coupled to, and the selection of these target classes reveals the specific member accesses the coupling consists of.

\section{RELATED WORK}

CouplingViz is intentionally narrowly focused on the visualisation of coupling as defined by the $\mathrm{CBO}$ metric. This distinguishes it from other tools which present an overview of multiple metrics simultaneously in order to gain insight into the system on many dimensions at once. It is believed that coupling is part of the intrinsic structure of a system and has more significance than that of a simple metric. The purpose of CouplingViz is to allow the interactive investigation of this significance. The use of a single metric also means that the representations of individual classes are relatively compact and allows large systems to be displayed in a similar fashion to the course-grained polymetric 2D views generated by CodeCrawler (Lanza, 2004). However, in the latter the classes are ordered according to one of the metrics under investigation and their positions do not relate to their location within the system. A fixed area allocated for each class in CouplingViz is a little less spaceefficient than CodeCrawler. However it does allow the types of couples to collaborators across the system to be clearly discerned when an individual class is selected and provides consistency between fan-in and fan-out views.

Several tools (including CodeCrawler's finegrained views) represent systems as graph-like structures in which classes are the nodes, with various metrics encoded in their representations and structural and/or coupling relationships between classes are the edges (Erdemir et al., 2011; Risi and Scanniello, 2012; Hanakawa, 2007). These representations tend to be inappropriate for full system overviews and do not share our narrow focus on coupling.

A number of tools have used a city-metaphor for visualising software systems in which classes are depicted as 3D buildings (Steinbrückner and Lewerentz, 2010; Wettel et al., 2011). Perhaps the most similar to CouplingViz are (Langelier et al., 2005), which uses CBO as one of three metrics encoded in each building, represented as a change in building colour from blue to red and (Caserta et al., 2011) in which actual coupling lines are shown above the city. These tools share the visualisation of a system overview with CouplingViz, but present much additional information beyond coupling, tending to require more screen space and more resources than the lighter-weight $2 \mathrm{D}$ approach used by CouplingViz.

Another family of tools related to CouplingViz depict dependencies. Managing dependencies is important in software development to allow systems to be architected into distinct independent modules. While there is considerable overlap between the concept of coupling between objects and dependency between classes they are not the same thing. One class depends on another class if it refers to that class type anywhere within it, but a couple exists only if it uses that type to access a member of it. Coupling is therefore a subset of the dependencies of a class. Furthermore, dependency visualisation tools tend to focus on a higher level than individual classes, typically depicting dependencies between packages. These tools include IntelliJIDEA (JetBrains, 2011), STAN (Odysseus, 2011), the 


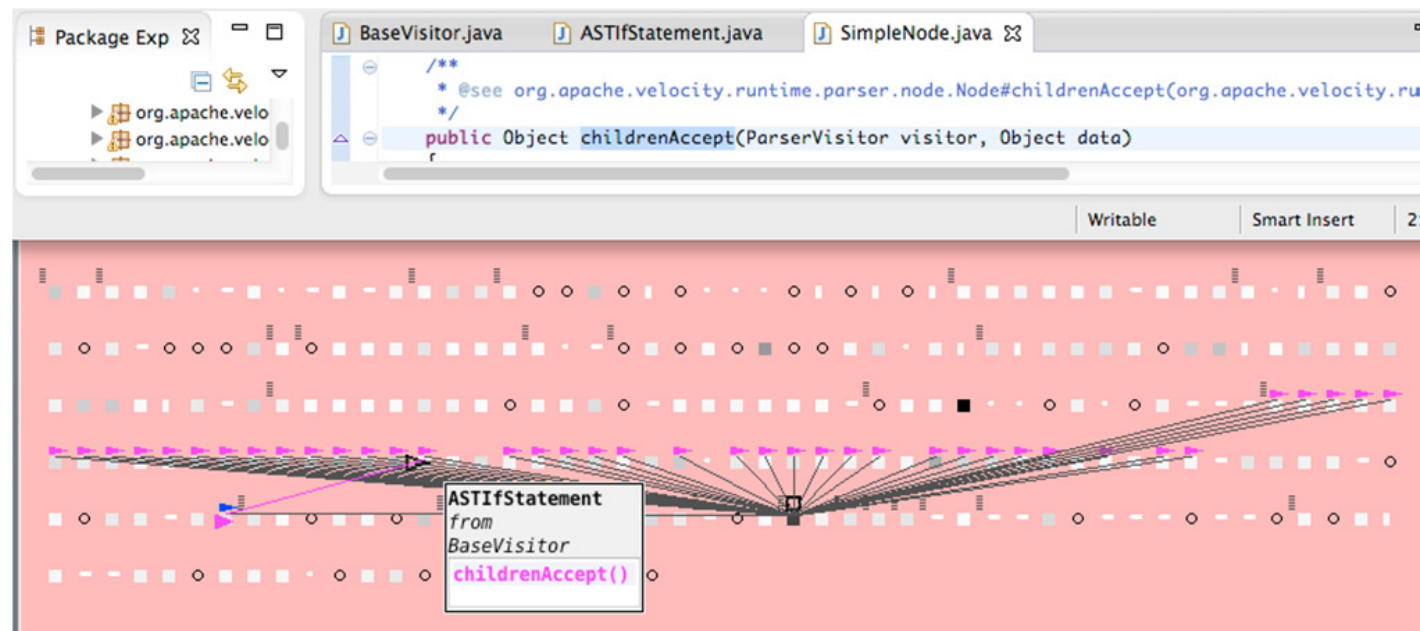

Figure 4: fan-out, foreign inheritance coupling BaseVisitor to ASTIfStatement.

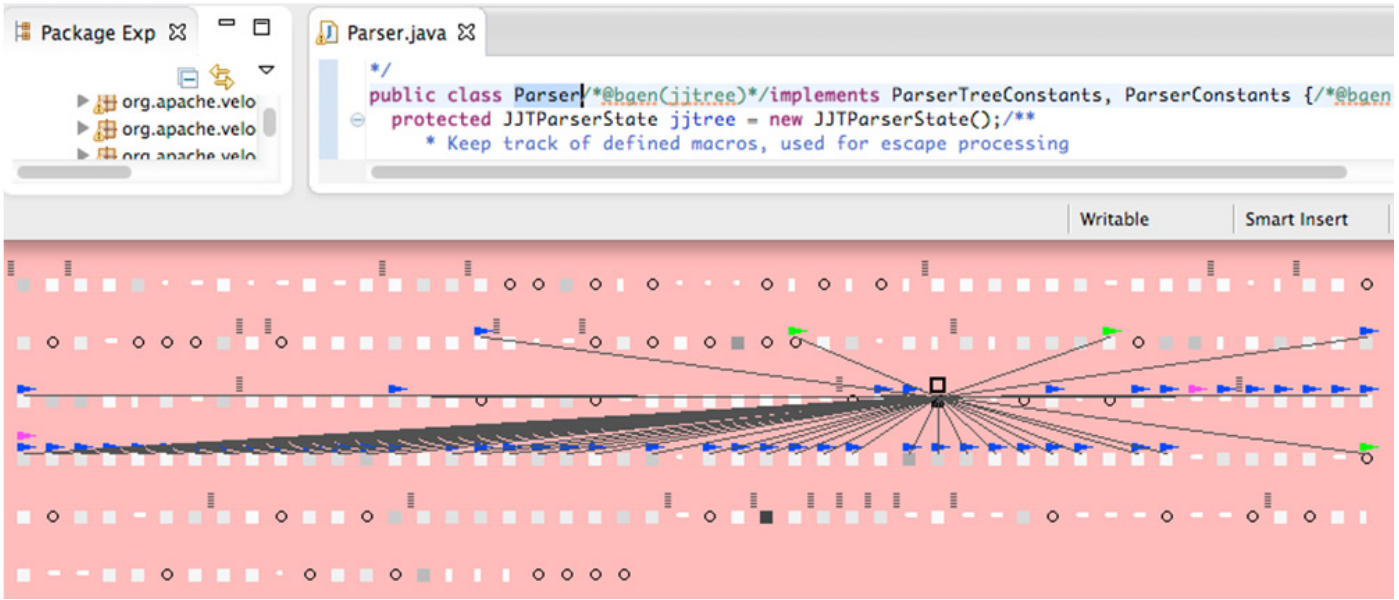

Figure 5: fan-out from Parser.

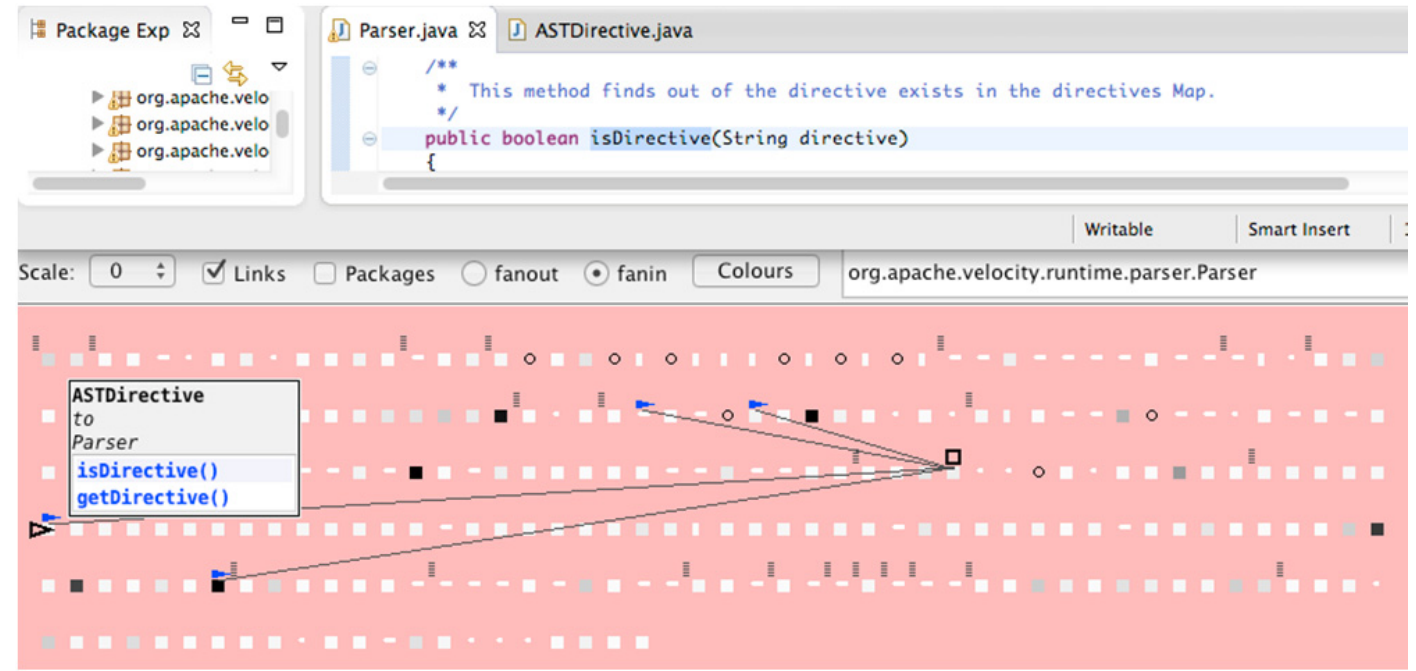

Figure 6: fan-in into Parser, method call isDirective() from ASTDirective to Parser selected. 
eDepend module of eUML2 (Soyatec, 2011), and Eclipse Metrics plug-in (Sauer, 2011). All but the last of these also enable drilling down to show the inter-class dependencies between two packages but none show an overview of dependencies for all classes in a system.

\section{COUPLING 'BY DESIGN' AND FAULT DATA}

Data for faults generated between releases 3.0.1 and 3.1.0 of the Eclipse JDT core project, mined for previous research (Counsell et al., 2013), was used for a pilot investigation of whether a developer can use a CouplingViz visualisation to successfully detect classes where high CBO coupling is not in fact harmful in terms of fault-proneness, addressing Q3b.

For this pilot investigation one of the authors of this paper, who was not given access to the fault data, analysed the Eclipse project using its CouplingViz visualisation. Classes with a CBO fanout of 20 or more were examined. The coupling was assessed in terms of any of the following: (1) package clustering of target classes, (2) repeated patterns of method invocations on many targets (3) indications from the category of coupling involved that a design pattern (such as factory or visitor) was present. Classes were categorised as showing evidence that a significant proportion of coupling resulted from such design features or else showing little or no such evidence. We called these categories design-coupled and ad-hoc coupled respectively, with the assumption that coupling arising from design is less harmful than in the general case. Correlation was then carried out using the fault data.

Figure 7 shows the distribution of the nine classes which were considered by the subject to be design-coupled. It shows the $\mathrm{CBO}$ of these nine classes and the faults that each of those classes exhibited between the two releases being considered. The fitted line is almost horizontal indicating a very low correlation between faults and CBO for these classes. The correlation value (Pearson's) was found to be just 0.02 . This is an interesting result since it shows that a class whose high coupling was designinspired is likely to contain fewer faults than we might expect (positively answering Q3b). The average number of faults for the set of nine classes was 14.11 and average CBO 90.44.
Figure 8 shows the corresponding graph for the classes considered by the subject to be ad-hoc coupled. There is a clear difference between the values in this figure and those in Figure 7. The correlation value was 0.20 in this case (not significant). The average number of faults for the set of sixty-five classes was 10.43 ; the average CBO was 31.31 .

The fact that for all the most highly coupled classes of this particular Java project there was no statistical significance to the correlation values of coupling against faults is unexpected. Despite this, the pilot study does indicate that a CouplingViz visualisation could be used to identify those classes with large amounts of coupling, but with a lesser propensity for faults than would otherwise be predicted. The work described thus provides some insight into a research problem that has been tackled very superficially until now - which types of coupling are harmful and which are relatively harmless? If this issue can be explored in depth, then guidelines can start to be formed on which types of coupling a designer can tolerate. CouplingViz thus provides, at a high level of abstraction, a means of observing and regulating that coupling, spotting dangerous trends and giving the developer information to tackle potential maintenance problems.

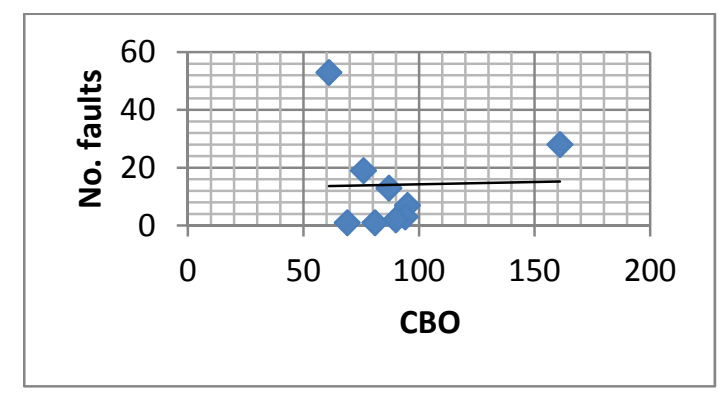

Figure 7: Eclipse JDT core, CBO vs faults, designcoupled' classes.

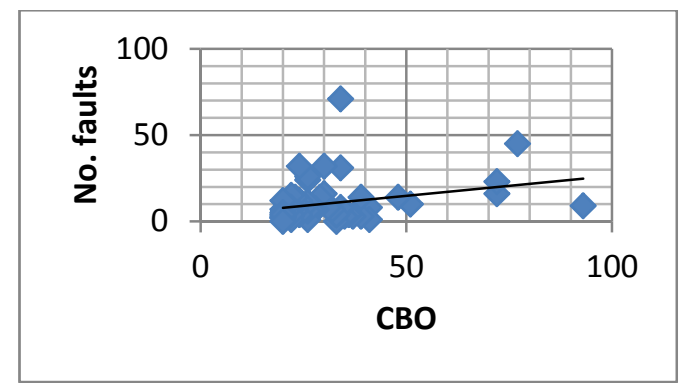

Figure 8: Eclipse JDT core, CBO vs faults - 'ad-hoc coupled' classes. 


\section{FUTURE WORK}

As well as developing the tool further, we plan to supplement the pilot study described above with more in-depth studies based on more developers. We also envisage using a wider sample of class sets, in order to validate the preliminary conclusions made.

\section{REFERENCES}

Biddle, R., Noble, J., Tempero, E., "Reflections on CRC Cards and OO Design," 40th International Conference on Technology of Object Oriented Languages and Systems (TOOLS Pacific), Sydney Australia, 2002.

Caserta, P., Zendra, O. and Bodenes, D. (2011) 3D Hierarchical Edge bundles to visualize relations in a software city metaphor, in: Visualizing Software for Understanding and Analysis (VISSOFT), 2011 6th IEEE International Workshop on, , pp. 1-8.

Chidamber, S. R., Kemerer, C. F., "A Metrics Suite for Object Oriented Design," IEEE Transactions on Software Engineering, 20(6). pp. 476-493, 1994.

Counsell, S., Gatrell, M., Hierons, R., Murgia, A., Tonelli, R., Marchesi, M., Concas, G., "Conditionalbased refactorings and fault-proneness: an empirical study," TAIC PART 2013.

Doernenburg, E., "Get the 1000-Foot View," in 97 Things Every Software Architect Should Know: Collective Wisdom from the Experts, p. 56, ed Monson Haefel, R., O’Reilly Media, Feb 2009.

Erdemir, U., Tekin, U. and Buzluca, F., "E-Quality: A graph based object oriented software quality visualization tool," Visualizing Software for Understanding and Analysis (VISSOFT), 2011 6th IEEE International Workshop, pp. 1-8, 2011.

Hanakawa, N. (2007) Visualization for Software Evolution Based on Logical Coupling and Module Coupling, in: Software Engineering Conference, 2007. APSEC 2007. 14th Asia-Pacific, pp. 214-221.

JetBrains Inc., IntelliJIDEA, 2011 [Online]. Available from: http://www.jetbrains.com/idea, Accessed 2013.

Langelier, G., Sahraoui, H. and Poulin, P., "Visualization-based analysis of quality for largescale software systems," Proceedings of the 20th IEEE/ACM international Conference on Automated software engineering, Long Beach, CA, USA, New York, NY, USA: ACM, pp. 214-223, 2005.

Lanza, M., "CodeCrawler - Polymetric views in action," Proceedings. In 19th International Conference on Automated Software Engineering: pp. 394-395, 2004.

Odysseus Software GmbH, STAN. [Online]. Available from: http://stan4j.com, 2011 [Accessed 2013].
Olague, H. M., Etzkorn, L. H., Gholston, S., Quattlebaum, S., "Empirical Validation of Three Software Metrics Suites to Predict Fault-Proneness of Object-Oriented Classes Developed using Highly Iterative or Agile Software Development Processes," IEEE Transactions on Software Engineering, 33(6): pp. 402-419, 2007.

Risi, M. and Scanniello, G., "MetricAttitude: a visualization tool for the reverse engineering of object oriented software," Proceedings of the International Working Conference on Advanced Visual Interfaces, Capri Island, Italy, New York, NY, USA: ACM, pp. 449-456, 2012.

Shneiderman, B., "The eyes have it: a task by data type taxonomy for information visualizations," Proceedings IEEE Symposium on Visual Languages, Boulder, Colorado, 3-6 September 1996, pp. 336343, 1996.

Sauer, F., Eclipse metrics plug-in [Online]. Available from: http://metrics.sourceforge.net, 2011. Accessed 2013.

Soyatec, eDepend, 2011 [Online]. Available from: http://www.soyatec.com/euml2 [Accessed 2013].

Steinbrückner, F. and Lewerentz, C., "Representing development history in software cities," Proceedings of the 5th international symposium on Software visualization, Salt Lake City, Utah, USA, New York, NY, USA: ACM, pp. 193-202, 2010.

Tufte, E. R., Envisioning information, Cheshire, Conn.: Graphics Press, 1990.

Wettel, R., Lanza, M. and Robbes, R., "Software systems as cities: a controlled experiment," Proceedings of the 33rd International Conference on Software Engineering, Waikiki, Honolulu, HI, USA, New York, NY, USA: ACM, pp. 551-560, 2011. 